Revista de Psicología Vol. 34 (2), 2016 (ISSN 0254-9247)

\title{
Descripción de las conductas de salud en un grupo de estudiantes universitarios de Lima
}

\author{
Silvia Becerra ${ }^{1}$ \\ Pontificia Universidad Católica del Perú
}

La presente investigación tuvo como objetivo analizar y describir las prácticas o conductas de salud que presentan un grupo de estudiantes en sus primeros años de estudio de una universidad privada de Lima. Participaron 155 estudiantes con promedio de edad de 18 años. Se les aplicó en forma anónima un cuestionario sobre conductas de salud. Los resultados indican que la mayoría de los alumnos presentan conductas saludables respecto a las áreas investigadas pero que paralelamente existen porcentajes significativos de alumnos que están presentando algunas conductas de riesgo, como la poca o ninguna realización de actividad y ejercicio físico (especialmente en el grupo de mujeres y los mayores), escaso o nulo cuidado preventivo de su salud, poco consumo de agua, frutas y verduras, consumo excesivo de alcohol y consumo de tabaco. Resalta la presencia, en un grupo considerable de alumnos, de problemas sintomáticos en el hábito del sueño y del descanso. Se recomienda la realización de programas de intervención en los estudiantes con el fin de informar, motivar y modificar sus conductas adversas.

Palabras clave: conductas de salud, estudiantes universitarios.

\section{Description of health behaviors in a group of university students from Lima}

The present study aimed to analyze and describe the health behaviors or practices that of students in their first years of study at a private university in Lima. One hundred fifty five students participated with an average age of 18 years. A measure of health behaviors was administered anonymously. The results indicate that most students engage in healthy behaviors, but there are significant percentages of students who are presenting some risk behaviors, such little or no physical activity and exercise (especially in the group of women and older students), little or no preventive health care, low consumption of water, fruits and vegetables, excessive alcohol consumption and excess use of tobacco. This study highlights the presence of symptomatic problems in sleep and leisure time in a large group of students. Intervention programs should be implemented in the university population to educate, motivate and change their adverse behaviors.

Keywords: Health behaviors, college students.

1 Magíster en Psicología Clínica de la Salud y docente a tiempo parcial por asignaturas por la Pontificia Universidad Católica del Perú. Dirección postal: Apartado 1761 Lima 100. Contacto: sbecerra@pucp.pe 


\section{Descrição das condutas de saúde em um grupo de estudantes universitários de Lima}

A presente pesquisa tem como objetivo analisar e descrever as práticas ou comportamentos relacionados à saúde que apresentam um grupo de estudantes em seus primeiros anos de estudo em uma universidade privada em Lima. Participaram 155 estudantes; a idade média foi de 18 anos. De forma anónima realizaram um teste sobre sua conduta em relação à saúde. Os resultados indicam que a maioria dos alunos apresentam condutas saudáveis em relação às áreas pesquisadas, mas, paralelamente, existem percentagens significativas de alunos que estão apresentando algumas condutas de risco, como pouca ou nenhuma realização de atividades ou exercícios físicos, (especialmente no grupo de mulheres e maiores), assim como pouco ou nenhum cuidado preventivo com sua saúde, pouco consumo de água, frutas e verduras, e consumo excessivo de álcool e consumo de tabaco. É importante destacar a presença, em um número considerável de alunos, de problemas sintomáticos no hábito do sono e do descanso. É recomendável a realização de programas de intervenção nos estudantes, com o fim de informar, motivar e modificar suas condutas adversas.

Palavras-chave: condutas em relação à saúde, estudantes universitarios. 
El estudio del estilo de vida o conductas de salud cobra una importancia significativa en la actualidad debido a los cambios que se han dado en los últimos ańos en las principales causas de morbimortalidad en la población mundial. Según un informe de la Organización Mundial de la Salud (Organización Mundial de la Salud [OMS], 2002), en los países industrializados estas causas han pasado de ser las referidas a trastornos infecciosos, tuberculosis, gripe, sarampión y poliomelitis por otros trastornos cuyas causas son prevenibles y directamente relacionadas con lo que los individuos hacen o dejan de hacer en la mayor parte de su vida, como el cáncer al pulmón, las enfermedades cardiovasculares, el abuso del alcohol y otras sustancias y los accidentes automovilísticos.

El Perú, a pesar de ser un país en vías de desarrollo, no es ajeno a esta realidad. Según informes epidemiológicos, el Perú se encuentra en una fase postransicional, lo que significa que la mayor causa de muertes en el país es debido a enfermedades no transmisibles (principalmente enfermedades cardio y cerebrovasculares, diabetes, cáncer y enfermedades mentales) y a las lesiones accidentales e intencionales (Valdéz, Ramos, Miranda \& Tovar, 2010). Algunos autores señalan que estas dolencias y muertes podrían ser sustancialmente reducidas alterando ciertos hábitos que se constituyen como factores de riesgo (Oblitas, 2010; Taylor, 2007). Es así que la relevancia del estilo de vida, expresado a través de las conductas de salud, en el estatus de salud es algo evidente y por ello se hace prioritario para las políticas de salud de un país el tener como objetivo la promoción de conductas saludables.

A nivel empírico, solo se ha demostrado que las conductas o comportamientos son los que tienen una influencia directa en la salud, el bienestar y la calidad de vida de una persona (OMS, 2002; Salazar, Varela, Lema, Tamayo \& Duarte, 2010). Es por esta razón que bajo 
un enfoque pragmático, se define el estilo de vida como pautas de comportamiento cuyo efecto es la promoción de la salud o la potenciación de los riesgos (Oblitas, 2010).

El estilo de vida suele ser definido como la manera o forma cómo la persona vive su vida, que incluye sus creencias, expectativas, motivos, valores y conductas (OMS, 2002). De todos estos componentes, solo se ha demostrado empíricamente que son las conductas las que tienen una influencia directa en la salud, el bienestar y la calidad de vida de las personas (Salazar, Varela, Lema, Tamayo \& Duarte, 2010). Es por esta razón, que se suele definir también al estilo de vida, como ciertas pautas de comportamiento cuyo efecto es la promoción de la salud o la potenciación de los riesgos (Oblitas, 2010).

Cuando este estilo de vida lleva a la preservación de la salud se habla de un estilo de vida saludable. Entre las conductas que componen un estilo de vida saludable, encontramos los que se relacionan con la actividad y ejercicio físico; los hábitos de alimentación; el consumo de tabaco, alcohol y otras drogas; la recreación y el manejo del tiempo libre; la prevención de enfermedades médicas y accidentes y los hábitos de sueño (Arrivillaga, Salazar y Correa, 2003).

Por otro lado, podemos afirmar que el inicio de la vida universitaria marca un hito importante en la historia de desarrollo de un individuo. En términos de edad coincide con los años de la adolescencia tardía y el inicio de la adultez temprana (17 años hasta los 25 o un poco más de años, usualmente). Se espera que al finalizar esta etapa el individuo haya logrado no solo un completo desarrollo físico y cognitivo, sino que esté próximo (o al menos en camino) de lograr su autonomía o madurez en sentido emocional, social y económico (Berger, 2001, 2007). El logro de esta importante tarea de desarrollo lo va a llevar a ser un adulto maduro y capaz de asumir la responsabilidad de cuidarse y cuidar a otros significativos, en un amplio sentido de la palabra. Esto implica que llegue a adoptar un estilo de vida saludable y a implementar conductas en pro de mantener un buen estado físico y salud general. Por ende, es crucial prestar atención a esta etapa de vida debido a que si bien, es referida por diversos autores del desarrollo humano como una 
etapa en el que gozamos del mejor estado de salud, es durante esta que se desarrollan e instalan patrones de comportamiento relacionadas a la salud, sean beneficiosos o de riesgo (Santrock, 2007).

En los estudiantes universitarios, se han realizado varias investigaciones a nivel internacional y también en Latinoamérica respecto a las conductas relacionadas a la salud que generalmente están presentes en ellos (American College Health Association - ACHA, 2011; Alonso, Pérez, Alcalá, Lubo y Consuegra, 2008; Grupo de Opinión Pública de la Universidad de Lima, 2006; Lema et al., 2009; Meda et al., 2008; Páez \& Castaño, 2010; Salazar \& Arrivillaga, 2004). Los resultados son amplios y muy diversos. En una vasta investigación realizada a nivel nacional con 30,093 estudiantes universitarios en Estados Unidos por la American College Health Association (ACHA, 2011), se encontró que si bien el $92 \%$ de los estudiantes de educación superior percibían su salud como buena, muy buena o excelente, esta percepción no coincidía con los principales hábitos de salud encontrados. Así, se observó que el 24\% de los estudiantes de educación superior no hace ejercicios; el $61.2 \%$ consume 1 o 2 veces por día frutas y vegetales; el 59.8\% ha usado alcohol, el 14.9\% fuma cigarrillos, el $14.2 \%$ marihuana y el $13.5 \%$ otras drogas en los últimos 30 días y, por último, el $90.5 \%$ tiene un problema con la somnolencia durante sus actividades diarias.

Con respecto a los resultados de estudios realizados en Latinoamérica, en México Meda et al. (2008) encontraron en una muestra de 405 estudiantes del Centro Universitario de Ciencias de la Salud de Guadalajara, que el $75 \%$ percibe su salud entre buena y muy buena y que la mayoría cuida de su salud. Sin embargo, resaltan que existen porcentajes significativos de estudiantes que declaran realizar ciertas conductas de riesgo para su salud, como no hacer alguna actividad física (el $23 \%$ de la muestra), fumar (27\%) y tener relaciones sexuales sin protección (15\%).

A su vez, en Colombia se han realizado varias investigaciones. Lema et al. (2009) evaluaron a 598 estudiantes de una universidad privada, encontrando que existe un predominio de prácticas saludables 
en todas las dimensiones evaluadas con excepción de ejercicio y actividad física. Más bien, Alonso et al. (2008) en una investigación con 540 estudiantes universitarios encontraron que las conductas de riesgo son prevalentes entre ellos pues solo un bajo porcentaje realiza actividad física moderada y vigorosa y que su dieta es pobre en frutas y vegetales y rica en carbohidratos y lípidos. Páez y Castaño (2010) en un estudio realizado con una muestra de 172 alumnos universitarios colombianos encontraron la presencia de algunas conductas de riesgo que podrían constituir factores de riesgo para la salud, como la presencia de problemas y dependencia al alcohol, práctica no regular de protección versus una enfermedad de transmisión sexual y un embarazo, alimentación mayormente inadecuada, actividad física insuficiente y sedentarismo. Salazar y Arrivillaga (2004) en un estudio más amplio realizado con 4416 estudiantes en una universidad de Colombia, reportan que el $95 \%$ tiene en general un estilo de vida saludable o muy saludable pero los hallazgos específicos en la dimensión consumo de alcohol, tabaco y otras drogas muestran un estilo de vida poco saludable, lo que indicaría que los jóvenes están incurriendo en prácticas de riesgo.

Finalmente, en relación con los estudios realizados en el Perú, cabe mencionar los resultados encontrados por el Grupo de Opinión Pública de la Universidad de Lima según una encuesta realizada en el 2005 a 1256 estudiantes universitarios pertenecientes al Consorcio de Universidades (Grupo de Opinión Pública de la Universidad de Lima, 2006). En cuanto a la actividad física, ellos encuentran que el $46.5 \%$ de los estudiantes no practica algún deporte y que el 46\% de los que sí lo practican lo hace 1 a 2 veces por semana (por debajo de los requerimientos recomendados). En cuanto a la salud nutricional, encuentran que los jóvenes tienen un bajo consumo de frutas y verduras y un alto consumo de proteínas y calorías, lo que sumado al sedentarismo, los podría poner en riesgo de ser obesos. Por último, en cuanto a su estado de salud, el $78.6 \%$ de la muestra considera su salud como buena y muy buena, sin embargo contradictoriamente se encontró que el 37.3\% fuma cigarrillos, el $63.1 \%$ consume bebidas alcohólicas y el $4.9 \%$ consume otras drogas. 
Como se puede observar, en general las investigaciones realizadas sobre el tema de las conductas de salud de los estudiantes universitarios concluyen que porcentajes variables según la conducta implicada, pero significativos, presentan conductas de riesgo que si se mantienen a largo plazo podrían llevarlos a desarrollar enfermedades crónicas y, eventualmente, a la muerte (Rodríguez, Ríos, Lozano \& Alvarez, 2009). Estas conductas se refieren principalmente a la inactividad física, al consumo de alimentos no saludables y al consumo de tabaco y bebidas alcohólicas en forma excesiva.

Como Posada-Lecompte (como se cita en Páez \& Castaño, 2010) afirma, el ingreso al ambiente universitario cambia actitudes y rutinas, se modifican los hábitos y el estilo de vida; es así que surgen actividades y hábitos que pueden afectar su calidad de vida y su salud en el futuro. Es por ello que se hace imperativo que las instituciones de educación superior promuevan la educación para la salud mediante programas de carácter multidisciplinario con el fin de hacer que los jóvenes se responsabilicen del papel activo que deben desempeñar en la promoción de su salud. Es importante motivarlos hacia la construcción de un proyecto de vida saludable como parte de su formación académica (Rodríguez et al., 2009).

Sobre la base de lo expuesto es que se planteó como objetivo de esta investigación, analizar y describir las prácticas o conductas de salud que presenta un grupo de estudiantes en sus primeros años de estudio de una universidad privada de Lima. Los resultados pueden aportar valiosa información que ayude posteriormente a promover hábitos adecuados de salud entre los estudiantes y fomente su salud integral, objetivo prioritario propuesto por el Ministerio de Salud en su resolución ministerial 968-2010/MINSA, bajo el lema "Promoviendo Universidades Saludables” (Ministerio de Salud [MINSA], 2010a). 


\section{Método}

\section{Participantes}

En la presente investigación participaron en forma voluntaria 155 estudiantes en sus primeros años de estudio de una universidad privada de Lima. Su promedio de edad fue de 18 ańos, el 59\% fueron mujeres y un $10,3 \%$ trabajaba, mayormente como profesores. El $77.1 \%$ ha nacido en Lima, un $19 \%$ en provincia (en su mayoría en la Sierra) y un $3.9 \%$ en el extranjero. El $65.2 \%$ de estudiantes ha presentado por lo menos una enfermedad en el último año, siendo las quejas o motivos reportados más frecuentes la tensión en el cuello y/o espalda, los dolores de espalda y cabeza y las molestias estomacales (41.9\%, 39.4\%, $25.8 \%$ respectivamente). Le siguen, en ese orden, los problemas de ansiedad (24.5\%), enfermedades respiratorias (15.5\%), depresión (12.3\%), insomnio (9.7\%) y migrańas (9\%). La media de los índices de masa corporal (IMC) tanto de hombres como de mujeres se encuentra, según las tablas de la OMS, dentro de los límites de un peso normal (IMC entre 18 y 24); sin embargo, un 5.3\% de la muestra se encuentra por debajo de este peso y un $16 \%$ presenta sobrepeso. Finalmente observamos que la mayoría de los estudiantes percibe actualmente su salud como buena $(54.2 \%)$, muy buena $(31 \%)$ o excelente $(5.8 \%)$.

\section{Medición}

Las conductas de salud se evaluaron a través de la primera parte del Cuestionario de Estilos de Vida en Jóvenes Universitarios-CEVJU elaborado por Arrivillaga, Salazar y Correa (2003) y reformulada para fines de esta investigación en conjunto con el investigador Juan Carlos Saravia (Becerra, 2013; Saravia, 2013).

Este instrumento implica una modificación del cuestionario original del CEVJU, en base a una revisión cuidadosa de cada ítem con la consulta de jueces expertos en cada tema o área. La versión resultante fue sometida a una prueba piloto con cuatro jóvenes de características similares a la población objetivo, que sirvió de ayuda para reformular la redacción de algunos ítems. 
La versión final del Cuestionario de Conductas de Salud consta de 65 ítems repartidos en seis áreas: actividad física y deporte (ítems 1-6); recreación y manejo del tiempo libre (ítems 7-10); autocuidado y cuidado médico (ítems 11-28); hábitos alimentarios (ítems 29-45); consumo de alcohol, tabaco y otras drogas (ítems 46-56) y organización del sueño y descanso (ítems 57-65). Las opciones de respuestas utilizan un formato tipo likert con cuatro alternativas: nunca, algunas veces, con frecuencia y siempre. El puntaje total por áreas se obtiene inviertiendo los ítems 2 , $10,17,18,26,27,30,31,32,33,36,37,38,42,43,44,45,46,47,49$, $50,51,54,55,56,59,60,61,63,64$ y 65 y después sumando todos los ítems de cada área. Los puntajes superiores son indicadores de que la persona posee buenos hábitos o conductas saludables y los inferiores, de que la persona posee pobres hábitos y por lo tanto, conductas de riesgo para su salud. Los alfa de Cronbach de las áreas variaron entre 0.47 y 0.75 .

\section{Procedimiento}

Esta investigación se realizó en una sola aplicación en las aulas de clase. A los estudiantes de cada aula, se les explicó la naturaleza e importancia de la investigación. Los que no desearon participar tuvieron la oportunidad de retirarse y los que accedieron a hacerlo, firmaron un consentimiento informado que les indicaba las condiciones de investigación y sus derechos como participantes de la misma, llenaron una hoja de datos demográficos y de salud y respondieron al cuestionario sobre conductas de salud.

Los datos obtenidos fueron procesados en el paquete estadístico SPSS-20. En primer lugar se analizó la confiabilidad del instrumento buscando su consistencia interna en base al alfa de Cronbach para ver si las áreas se organizaron coherentemente. Luego se procedió a utilizar estadísticos descriptivos como la distribución de frecuencias y medidas de tendencia central para analizar los datos y características de los sujetos de la muestra. Finalmente, se obtuvieron las diferencias estadísticas entre las variables demográficas y de salud pertinentes y las conductas de salud. Esto se realizó utilizando el estadístico $U$ de Mann Whitney, cuando las variables constaban de dos grupos, y el $H$ de Kruskal-Wallis, cuando estas estaban constituidas por tres grupos. 


\section{Resultados}

En relación con las conductas de salud, en la Tabla 1 se pueden observar los resultados a nivel descriptivo.

\section{Tabla 1}

Descripción de los puntajes según conductas de salud

\begin{tabular}{lccc}
\hline Variable & $M d n$ & $M$ & $D E$ \\
\hline Conductas de salud & 12 & 13.0 & 3.4 \\
$\quad$ Actividad física y deporte & 12 & 11.6 & 2.0 \\
Recreación y manejo del tiempo libre & 44 & 43.6 & 4.9 \\
Autocuidado & 45 & 45.2 & 6.0 \\
Hábitos alimentarios & 33 & 32.8 & 4.7 \\
Consumo de alcohol, tabaco y otras drogas & 25 & 25.5 & 3.0 \\
Organización del sueño y descanso & & & \\
\hline
\end{tabular}

$\mathrm{n}=155$

Al realizar un análisis de las áreas de las conductas de salud, se aprecia, con respecto a actividad física y deporte, que si bien el $85 \%$ de la muestra incluye la realización de actividad física en su vida cotidiana algunas veces, con frecuencia o siempre, solo un $40 \%$ realiza ejercicios físicos o un deporte en forma regular y un $16 \%$ lo hace utilizando pesas. Muy pocos $(7.1 \%)$ realizan ejercicios que les ayuden a estar tranquilo y solo el $29.7 \%$ de ellos termina el día sin un cansancio excesivo con frecuencia o siempre.

Con relación al área de recreación y uso del tiempo libre, se observa que aproximadamente las tres cuartas partes de los participantes incluye momentos de descanso en su rutina diaria, comparte con su familia y/o amigos su tiempo libre y realiza actividades placenteras en su tiempo libre con frecuencia o siempre. Sin embargo, cerca de la mitad de la muestra destina parte de su tiempo libre para actividades académicas con frecuencia o siempre. 
Respecto al área de autocuidado y cuidado médico, se observa en general que entre el 50 y el $75 \%$ de los participantes presenta una nula o pobre conducta preventiva de su salud física, como ir al dentista o al médico una vez al año, evitar exposiciones prolongadas al sol o usar protector solar y revisar su cuerpo con detenimiento para detectar algún cambio físico (manchas, bultos). Solo el $13.6 \%$ revisa al menos una vez al año su presión arterial y el $19.4 \%$ no se automedica en caso de dolores musculares y resfriados con frecuencia o siempre, y el $58.7 \%$ lee y sigue instrucciones cuando utiliza algún medicamento con esta misma frecuencia. Entre las mujeres, el $94.6 \%$ nunca se ha realizado un papanicolau. No obstante, por otro lado, el $80 \%$ o más de la muestra tiene una buena conducta preventiva vial, como obedecer las reglas de tránsito, no manejar ni abordar un vehículo manejado por un conductor bajo los efectos del alcohol u otras drogas y usar el cinturón de seguridad.

En relación con el área de hábitos alimentarios, se aprecia que alrededor del $50 \%$ de los participantes presenta, con frecuencia o siempre, hábitos saludables como tomar entre 4 a 8 vasos de agua al día, no añadir ni sal ni azúcar a sus comidas y bebidas y no consumir bebidas artificiales o dulces más de dos veces por semana. A pesar de que solo el $29.6 \%$ de ellos come con frecuencia o siempre cinco porciones de frutas o verduras diarias, un porcentaje que va entre el 50\% y el $75 \%$ de la muestra come más pescado y pollo que carnes rojas, limita sus consumos de grasa, embutidos, snacks no saludables y comidas rápidas. Por otro lado, si bien casi el $60 \%$ no mantiene un horario regular en sus comidas, muy pocos dejan de comer aunque tengan hambre o comen en exceso y realizan dietas sin supervisión médica.

Respecto al área de consumo de alcohol, tabaco y otras drogas, se observa que un $65.8 \%$ de la muestra no fuma tabaco y los que sí lo hacen, solo el $9.7 \%$ (del total) fuma más de media cajetilla de cigarrillos a la semana. En cuanto al consumo de alcohol, un $24.5 \%$ toma algunas veces más de dos veces a la semana y un $4.5 \%$ lo hace con frecuencia y siempre. De todos los hombres, un $50.8 \%$ bebe con frecuencia o siempre cinco o más vasos o copas de alcohol cada vez que toma; 
en el caso de las mujeres este porcentaje se reduce a 30.4\% (tres o más vasos o copas). Sin embargo, un $76.1 \%$ de la muestra afirma que con frecuencia o siempre pueden reconocer en qué momento deben parar cuando empiezan a beber. Respecto al consumo de otras drogas, un $11.6 \%$ reconoce que las consume algunas veces y un $2.6 \%$ siempre. En general, el $77.4 \%$ niega consumir algún tipo de drogas cuando se enfrenta a problemas en su vida.

Finalmente, en cuanto al área de la organización del sueño y el descanso, un 58\% duerme al menos siete horas diarias pero solo el $36.8 \%$ duerme bien y se levanta descansado con frecuencia o siempre, habiendo un $85.2 \%$ que presenta somnolencia durante el día alguna veces, con frecuencia o siempre. Un $66.6 \%$ trasnocha entre algunas veces y siempre, a un $66.5 \%$ le cuesta trabajo quedarse dormido y un $61.3 \%$ se despierta durante la noche en varias ocasiones con la misma frecuencia. Muy pocos $(27.1 \%)$ tienen la costumbre de hacer siesta y solo un $6.4 \%$ está utilizando algunas veces o siempre pastillas para dormir. Finalmente se observa que el $41.9 \%$ algunas veces planea sus actividades respetando sus horarios de sueño, un $18.7 \%$ nunca lo hace y solo el $39.3 \%$ lo hace entre siempre o con frecuencia.

En lo concerniente a las diferencias significativas entre los datos demográficos y de salud y las conductas de salud, se observa que se encuentran diferencias significativas en el sexo, año de estudio, presencia de enfermedad en el último ańo, el IMC y la percepción de salud. En general los hombres $(U=2002.50, p<0.01)$, los que están cursando su primer ańo de estudio $(U=1704.00, p<0.05)$, los que no han presentado una enfermedad en el último ańo $(U=2094.50, p<0.05)$ y los que perciben su salud como excelente y muy buena $(H=22.39$, $p<0.01)$ tienen mejores hábitos de salud respecto al área de actividad física y deportes; mientras que las mujeres lo tienen en el consumo de alcohol, tabaco y otras drogas $(U=2325.00, p<0.05)$. En cuanto al IMC, se encuentra que los que tienen un peso por debajo de lo normal presentan mejores hábitos alimentarios en comparación a los que están con sobrepeso o tienen un peso dentro de lo normal $(H=6.54$, $p<0.05)$. 


\section{Discusión}

La adolescencia es un periodo de grandes cambios a nivel biológico, social, cognitivo y emocional por los cuales el adolescente tiene que transitar y lograr adaptarse, con el fin de adquirir las habilidades necesarias para llegar a ser un adulto responsable y autónomo. Las investigaciones modernas han seńalado que esta no es necesariamente una etapa de conflictos y crisis en el sentido negativo, sino que mas bien es una etapa de retos y cambios que la mayoría de adolescentes suele pasar sin presentar problemas cruciales (Musiti, Martínez \& Varela, 2011; Santrock, 2007). Debido a las características propias de estos cambios, esta etapa y también la siguiente, la juventud, constituyen períodos claves y trascendentes para la adopción de comportamientos relacionados con la salud, entre los que se destacan la práctica del ejercicio físico, el consumo de alimentos considerados como no saludables, el consumo de tabaco, alcohol y otras drogas, las prácticas sexuales de riesgo, el realizar conductas violentas o imprudentes que ponen en riesgo su vida, entre otros (Berger, 2001, 2007; Santrock, 2006). Sin embargo, los jóvenes no perciben estos riesgos en forma realista pues se encuentran poco identificados con problemas de salud que suelen aparecer en edades posteriores (Santrock, 2007; Taylor, 2007). Por ello se hace necesario centrarse en el conocimiento y evaluación de las conductas referidas a la salud en la población joven.

Según el análisis de los resultados, es importante empezar comentando que en la mayoría de los alumnos, encontramos la presencia de conductas saludables respecto a las áreas investigadas: el 65\% de los estudiantes incluye la realización de actividad física en su vida cotidiana, cerca del $75 \%$ utiliza su tiempo libre para descansar o realizar actividades placenteras junto con su familia y/o amigos, el $80 \%$ muestra una buena conducta preventiva vial, el $66 \%$ no fuma tabaco, alrededor del $70 \%$ toma alcohol menos de dos veces por semana y cerca del $90 \%$ no consume drogas ilegales.

A pesar de esto, paralelamente se observa la presencia de porcentajes significativos de alumnos que están presentando algunas conductas de riesgo. 
En el área de actividad física y deporte, un 43\% no realiza actividad física en su vida cotidiana con una frecuencia adecuada y un $61 \%$ no está haciendo ejercicios físicos o un deporte en forma regular. Los resultados van en la misma línea de lo encontrado por investigaciones internacionales y nacionales sobre la realización de actividad física y deporte en estudiantes universitarios (Alonso et al., 2008; ACHA, 2011; Grupo de Opinión Pública de la Universidad de Lima, 2006; Lema et al., 2009; Meda et al., 2008). En el ámbito nacional, según una reciente encuesta realizada por Chau y Tavera (2012) en un grupo de 683 estudiantes universitarios de una universidad privada de Lima, se encuentra que el $51 \%$ realiza actividad física solo a veces o casi nunca. Esto puede llegar a ser preocupante debido a que existen investigaciones que resaltan la gran importancia que tiene la actividad física en la prevención de enfermedades como los trastornos cardiovasculares, diabetes, diversos tipos de cáncer, entre otras enfermedades (Fiorentino, 2010; Gil, 2004); en el fortalecimiento de la salud (Edenfield \& Blumenthal, 2011); en la reducción de la mortalidad y en una mayor longevidad (Córdova et al., 2001).

Asimismo, los resultados de esta investigación también nos señalan que son los hombres y los que están cursando su primer año de estudio, quienes presentan mejores hábitos relacionados a la actividad física. Esto concuerda con estudios realizados sobre actividad física y variables demográficas, los cuales resaltan que las mujeres, como grupo, hacen menos ejercicio físico que los hombres y que se encuentra una tendencia a reducir el nivel de ejercicio con la edad, especialmente a partir de la adolescencia (Córdova et al., 2001; Gil, 2004). También pueden indicarnos la dificultad que presentan los estudiantes de realizar actividad física debido a la interferencia que conlleva el aumento de la carga académica. Todos estos resultados indicarían la necesidad entonces, de promover el incremento del involucramiento de los estudiantes en la práctica del ejercicio físico, enfocándose principalmente en el grupo de mujeres y los que están en sus años intermedios y finales de su preparación. 
Con relación al área de autocuidado y cuidado médico, se encuentra que la mayoría no cuida preventivamente de su salud; esto se aprecia no solamente en actividades que personas de esta edad pueden percibir como no necesarias aún, como ir al médico, revisar su presión arterial o hacerse la prueba de papanicolau (en el caso de las mujeres) una vez al año, sino también en aspectos que sí los atañen, como ir al dentista una vez al ańo, evitar exposiciones prolongadas al sol o usar bloqueador cuando lo vayan a hacer y examinarse el cuerpo regularmente para detectar cambios físicos que podrían ser indicadores de la presencia de algún problema. Este hecho puede estar relacionado con la dificultad que los jóvenes presentan de relacionar sus conductas con consecuencias adversas anticipadas cuando estas no son visibles inmediatamente. Se ha demostrado que las conductas mencionadas están relacionadas con la prevención de enfermedades que pueden ser mortales, como el cáncer por ejemplo y, a largo plazo, pueden predisponer a estos jóvenes a sufrir dichas consecuencias si esta conducta no es modificada a tiempo. En el Perú, los tipos de cáncer más comunes en hombres son el de estómago, de pulmón y de próstata y, en las mujeres, son el de mama y de cuello uterino (Ministerio de Salud, 2010b). Estos tipos de cáncer y otros más como el de piel, podrían ser mayormente evitados si es que la persona fortaleciera sus conductas de autocuidado y realizara chequeos médicos en forma oportuna.

Respecto al área de hábitos alimentarios, entre un 50\% al 75\% no cumple con los requerimientos mínimos de una alimentación saludable en cuanto al consumo de agua o líquidos, uso de la sal y el azúcar en las comidas y consumo de frutas y verduras. Asimismo, se encuentran porcentajes variables que, aunque menores del $50 \%$, son igualmente indicadores de la presencia de grupos en riesgo, que no limita su consumo de grasa, embutidos, bebidas artificiales, bocadillos no saludables y comidas rápidas. Esto también ha sido encontrado por otras investigaciones, tanto nacionales (Chau \& Tavera, 2012; Grupo de Opinión Pública de la Universidad de Lima, 2006) como internacionales (Alonso et al., 2008; ACHA, 2011; Páez \& Castaño, 2010). Citando nuevamente la investigación realizada por Chau y Tavera (2012), 
ellas encontraron que solo el $50 \%$ de los estudiantes comen a veces cinco porciones de verduras y frutas y cerca del $30 \%$ casi nunca lo hace; además en su mayoría $(56,7 \%)$ consumen comida chatarra, bastante azúcar o grasa, aunque un 14,5\% consume todas esas opciones. Las posibles implicancias de estos inadecuados hábitos alimentarios son diversas, desde el estreñimiento crónico debido al déficit de un consumo suficiente de fibra; la presencia de caries dentales debido a la alta frecuencia y el tipo de azúcar consumido; la hipertensión debido al exceso del consumo de sal, cafeína y/o grasas saturadas; la arterioesclerosis debido al consumo excesivo de grasas saturadas y el cáncer, debido al consumo insuficiente de fibras, de frutas y verduras y a la excesiva ingesta también de grasas, entre otras razones (Gil, 2004). Como Labiano (2010) afirma: "del alimento que ingerimos a diario depende, en gran medida, el grado de bienestar que tenemos y también, cómo nos sentiremos en el futuro" (p. 300).

Es también importante considerar que un porcentaje de estudiantes participantes de la presente investigación, aunque pequeño, presenta problemas de bajo y sobrepeso (5.3\% y $16 \%$ respectivamente). Todo esto resalta la importancia de prestar atención a esta importante y básica conducta de salud, con el fin de trabajar con los hábitos alimentarios de los estudiantes.

En cuanto al consumo de alcohol, hábito difundido en nuestro medio y presente en los estudiantes universitarios (CEDRO, 2004; Chau, 2004, PRADICAN, 2013), observamos que si bien un 76\% de la muestra afirma saber cuándo parar de beber para no excederse, cerca del $51 \%$ de los varones y del $30 \%$ de las mujeres ingiere una cantidad de alcohol reconocida por los expertos en el tema como excesiva debido a que los pone en riesgo de lesión orgánica y riesgos objetivos para la salud (Córdova et al., 2001). Esto nos indicaría una tendencia en los jóvenes a sobreestimar su capacidad de control de la ingesta de alcohol. Con relación al consumo de tabaco, el 34.2\% de los estudiantes fuma cigarrillos y un $14.2 \%$ lo hace en una cantidad significativa. Esta cifra coincide con el reporte del consumo de tabaco en universitarios del Proyecto PRADICAN (2013), el cual señala que existe una prevalencia 
de consumo de $36.2 \%$ en esta población en el último año. Finalmente, en cuanto al consumo de drogas ilícitas, cerca del 15\% de los estudiantes reportaron haberlas consumido por lo menos ocasionalmente. Esto último es preocupante debido a la consideración de que el consumir alguna de estas sustancias puede ser motivo de ingreso al consumo de drogas más fuertes (CEDRO, 2004).

El incremento en la prevalencia del consumo de sustancias en nuestro medio y también el descenso de la edad de inicio (PRADICAN, 2013) nos hace prestar atención a esta importante conducta de salud. Las personas que consideran que su consumo no representa un serio peligro para la salud probablemente no tienen presente que los estudios muestran que el hecho de probar una droga ya pone al individuo en riesgo de insertarse en patrones más intensos de consumo que incluso podrían implicar adicción (CEDRO, 2004). Además de este lamentable descenlace, las investigaciones que dan cuenta de los efectos adversos sobre la salud del consumo de sustancias son bien documentadas y amplias, desde una variedad de cánceres, enfermedades cardiovasculares, daño pulmonar y a otros órganos del cuerpo, hasta la muerte. Además de contribuir a la enfermedad y al daño, el consumo excesivo de drogas también afecta a la salud debido a que conlleva a emitir conductas de riesgo, que incluyen prácticas de sexo no seguro, accidentes y violencia (Chau, 2004; Fiorentino, 2010; Grunberg, Shafer Berger \& Hamilton, 2011; Taylor, 2007).

Finalmente, se encuentra la presencia de problemas sintomáticos en el hábito del sueño y del descanso. Se observan, en un grupo considerable de alumnos, patrones de sueño disfuncionales, como trasnochar o no respetar los horarios de sueńo adecuados, lo cual está conllevando problemas de insomnio, como dificultades para quedarse dormido o despertarse varias veces durante la noche. Esto explicaría las razones por las que una mayoría de alumnos reporta no dormir y descansar bien, presentando consecuentemente problemas de somnolencia durante el día. Estos resultados son similares a los encontrados en la encuesta realizada por Chau y Tavera (2012), quienes reportaron que el $80 \%$ de los estudiantes universitarios manifestaron que solo a veces 
o casi nunca duermen bien y se sienten descansados. Esto podría estar relacionado con las demandas académicas y con patrones de actividad física y alimentación inadecuada. Una investigación realizada en estudiantes universitarios (Boullosa, 2013) encontró que la somnolencia, el cansancio permanente y las dificultades para dormir fueron las reacciones físicas más experimentadas por ellos debido a la percepción de las demandas académicas como estresores.

Asimismo, la Primera Encuesta Nacional de la Juventud realizada en el 2011 encontró que el síntoma relacionado a la salud mental más reportado en los jóvenes fueron los cambios en su ritmo de sueño (56.4\% de los jóvenes encuestados) (Secretaría Nacional de la Juventud [SENAJU], 2012). Estos datos evidenciarían la posible presencia de problemas de sueño en estos grupos de estudiantes universitarios y, en general, en los jóvenes peruanos. Es importante señalar que una buena calidad del sueño no solo constituye un factor determinante de la salud sino que además supone un elemento crucial en una buena calidad de vida (Grimaldo, 2010; Sierra, 2010). El sueño insuficiente afecta el funcionamiento cognitivo, el estado de ánimo y el desempeño en el trabajo; asimismo puede incrementar el riesgo de padecer una enfermedad coronaria o diabetes, reducir el funcionamiento inmune y predisponer a la persona a involucrarse en accidentes de tránsito (Taylor, 2007).

Los resultados obtenidos en la presente investigación constituyen una fuente válida que da sustento a la realización de programas de intervención en los estudiantes con el fin de informar, motivar y modificar sus conductas adversas, propiciando así el establecimiento y consolidación de hábitos saludables que los lleven a tener un buen estado físico y mental y una buena salud en general. Como psicólogos, poseemos herramientas útiles y efectivas para lograr el cambio de los comportamientos que no favorecen la salud; sin embargo, alcanzar o mantener estos cambios es un proceso complejo que requiere también de un enfoque que priorice al entorno como factor vital. Esto es lo que da fundamento para el trabajo, dentro del espacio universitario, bajo un enfoque de universidades saludables. 
Se recomienda, para futuras investigaciones, que se elabore un cuestionario específico a la población con la cual se quiera trabajar, con preguntas pertinentes en relación con la cultura en que está inmersa. Se sugiere además que se limite el número y el tipo de preguntas a las que se han encontrado relevantes para esta población. Por último, se propone la separación en dos del área de autocuidado y cuidado médico, una referida al cuidado preventivo de la salud física y la otra a la conducta preventiva vial.

\section{Referencias}

Alonso, L., Pérez, M., Alcalá, G., Lubo, A. \& Consuegra, A. (2008). Comportamientos de riesgo para la salud en estudiantes colombianos recién ingresados a una universidad privada en Barranquilla (Colombia). Salud Uninorte, 24(2), 235-247.

American College Health Association-ACHA (2011). National college health assessment. EEUU: American College Health Association. Arrivillaga, M., Salazar, I. \& Correa, D. (2003). Creencias sobre la salud y su relación con las prácticas de riesgo o de protección en jóvenes universitarios. Colombia Médica, 34(4), 186-195.

Becerra, S. (2013). Rol del estrés percibido y su afrontamiento en las conductas de salud de estudiantes universitarios de Lima (Tesis de maestría inédita). Pontificia Universidad Católica del Perú, Lima, Perú.

Berger, K. (2001). Psicología del desarrollo: adultez y vejez. Madrid: Médica Panamericana S.A.

Berger, K. (2007). Psicología del desarrollo: infancia y adolescencia. Madrid: Médica Panamericana S.A.

Boullosa, G. (2013). Estrés académico y afrontamiento en un grupo de estudiantes de una universidad privada de Lima (Tesis de licenciatura inédita). Pontificia Universidad Católica del Perú, Lima, Perú. 
CEDRO (2004). Estudio sobre drogas en universitarios de Lima. Recuperado de www.cedro.org.pe/ebooks/universitarios.pdf

Chau, C. (2004). Determinants of alcohol use among university students. The role of stress, coping and expectances (Tesis doctoral inédita). Katholieke Universiteit Leuven, Amsterdam, Bélgica.

Chau, C. \& Tavera, M. (2012). Informe proyecto PUCP-Saludable. I. Diagnóstico situacional. Manuscrito inédito. Lima: Pontificia Universidad Católica del Perú.

Córdova, R., Ortega, R., Cabezas, C., Forés, D., Nebot, M. \& Robledo, T. (2001). Recomendaciones sobre estilo de vida. Atención Primaria, 28(2), 37-50.

Edenfield, T. \& Blumenthal, J. (2011). Exercise and stress reduction. En R. Contrada \& A. Baum (Eds.). The handbook of stress science. Biology, psychology and health (pp. 301-319). New York: Springer Publishing Company.

Fiorentino, M.T. (2010). Conductas de la salud. En L. Oblitas (Coord.). Psicología de la salud y calidad de vida (pp. 57-81). México D.F.: Cengage Learning Editores S.A.

Gil, J. (2004). Alimentación, actividad física y salud. En J. Gil (Dir.), Psicología de la salud. Aproximación histórica, conceptual y aplicaciones (pp. 265-308). Madrid: Pirámide.

Grimaldo, M. (2010). Calidad de vida y estilo de vida saludable en un grupo de estudiantes de posgrado de la ciudad de Lima. Pensamiento psicológico, 8(15), 17-38.

Grunberg, N.; Shafer Berger, S. \& Hamilton, K. (2011). Stress and drug use. En R. Contrada y A. Baum (Eds.). The handbook of stress science. Biology, psychology and health (pp. 287-300). New York: Springer Publishing Company.

Grupo de Opinión Pública de la Universidad de Lima (2006). Perfil de los estudiantes del consorcio de universidades. Resumen y análisis. Lima: Consorcio de Universidades.

Labiano, M. (2010). Estrategias de mejoramiento de la calidad de vida. En L. Oblitas (Coord.), Psicología de la salud y calidad de vida (pp. 299-336). México D.F.: Cengage Learning Editores S.A. 
Lema, L., Salazar, I., Varela, M., Tamayo, J., Rubio, A. \& Botero, A. (2009). Comportamiento y salud de los jóvenes universitarios: satisfacción con el estilo de vida. Pensamiento Psicológico, 5(12), 71-87.

Meda, R., de Santos, F., Lara, B., Verdugo, J., Palomera, A. \& Valadez, M. (2008). Evaluación de la percepción de calidad de vida y estilo de vida en estudiantes desde el contexto de las Universidades Promotoras de la Salud. Revista de Educación y Desarrollo, 8, 5-16.

Ministerio de Salud-MINSA (2010a). Documento técnico promoviendo universidades saludables. Lima: Ministerio de Salud.

Ministerio de Salud-MINSA (2010b). El cáncer se previene y se puede curar si se detecta a tiempo. Recuperado de www.minsa.gob.pe/ portada/Especiales/2010/cancer/datos.aspv

Musiti, G., Martínez, B. \& Varela, R. (2011). El ajuste en la adolescencia: las rutas transitorias y persistentes. En R. Pereira (Comp.), Adolescentes en el siglo XXI. Entre impotencia, resiliencia y poder (pp. 109-128). Madrid: Morata.

Oblitas, L. (2010). Psicología de la salud y calidad de vida. México D.F.: Cengage Learning Editores S.A.

Organización Mundial de la Salud-OMS (2002). Informe sobre la salud en el mundo. Reducir los riesgos y promover una vida sana. Recuperado de: http://www.who.int/whr/2002/en/whr02_es.pdf

Páez, M. \& Castaño, J. (2010). Estilos de vida y salud en estudiantes de una facultad de psicología. Psicología desde el Caribe, 25, 155178.

Proyecto "Programa Anti-drogas Ilícitas en la Comunidad AndinaPRADICAN" (2013). II estudio epidemiológico andino sobre consumo de drogas en la población universitaria. Informe Perú, 2012. Lima: Secretaría General de la Comunidad Andina.

Rodríguez, N., Ríos, M., Lozano, L. \& Alvarez, M. (2009). Percepción de jóvenes universitarios respecto a su salud: conductas y contexto de riesgo. Enseñanza e Investigación en Psicología, 14(2), 245-260. 
Salazar, I. \& Arrivillaga, M. (2004). El consumo de alcohol, tabaco y otras drogas, como parte del estilo de vida de jóvenes universitarios. Revista Colombiana de Psicología, 13, 74-89.

Salazar, I., Varela, M., Lema, L., Tamayo, J. \& Duarte, C. (2010). Evaluación de las conductas de salud en jóvenes universitarios. Revista de Salud Pública, 12(4), 599-611.

Santrock, J. (2006). Psicologia del desarrollo, el ciclo vital. Madrid: McGraw-Hill/Interamericana de España, S.A.U.

Santrock, J. (2007). Adolescence. New York: McGraw-Hill.

Saravia, J. C. (2013). Factores psicológicos y conductuales de la salud en un grupo de universitarios de Lima Metropolitana (Tesis de maestría inédita). Pontificia Universidad Católica del Perú, Lima, Perú.

Secretaría Nacional de la Juventud (SENAJU) (2012). Primera encuesta nacional sobre la juventud. Lima: SENAJU-Ministerio de Educación.

Sierra, J.C. (2010). La calidad de sueño como factor relevante de la calidad de vida. En L. Oblitas (Coord.), Psicología de la salud y calidad de vida (pp. 337-344). México D.F.: Cengage Learning Editores S.A.

Taylor, S. (2007). Psicología de la salud. México D.F.: McGraw Hill.

Valdéz, W., Ramos, W., Miranda, J. \& Tovar, J. (2010). Análisis de la situación de salud del Perú. Lima: Ministerio de Salud. Dirección General de Epidemiología.

Recibido el 17 de noviembre, 2014 Aceptado el 08 de abril, 2016 